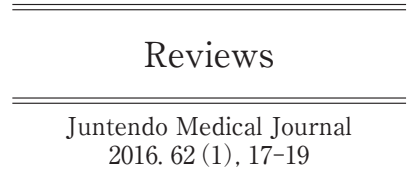

2016. $62(1), 17-19$

\title{
Fascinating Oligosaccharide Research
}

\author{
OSAMU HOSOMI* \\ *Graduate School of Health and Sports Science, Juntendo University, Chiba, Japan
}

\begin{abstract}
I had decided on my life plan of research when I left for my new position (Juntendo University). I gradually became interested in functional sugar chains in the body because the functions of saccharides (particularly, sugar chains and oligosaccharides) had not been elucidated, being considered as just decorations. However, antiinflammatory effects of glucosamine and chitosan had been described several decades earlier. I found a report on the anti-cancer effect of glucosamine on cancer tissue transplanted in mice in 'Nature', and this report gave me an opportunity to consider sugar function research. I repeatedly considered a new idea concerning glucosamine and animal lectin without telling anyone. If lectin existing in many animals and plants proved to be useful for this purpose, glucosamine would exhibit an anti-cancer effect, and the next target which came to my mind was the synthesis of a new sugar chain (galactose $\beta 1-4 / 6$ glucosamine, Gal $31-4 / 6 \mathrm{GlcNH}_{2}$ ).
\end{abstract}

Key words: oligosaccharide, glucosamine, lectin, cancer

Firstly, I would like to thank the Juntendo Medical Journal for giving me an opportunity to write this report. I served as a part-time lecturer at Juntendo University for 10 years (1993-2003), and was appointed as an assistant professor at Juntendo University Faculty of Health and Sports Science (Health Science) in April 2003. I had studied human blood types and sugar chains, particularly the sugar chain synthesis process and glycosyltransferase, and gradually became interested in functional sugar chains present in our body.

First, I realized that chitosan and glucosamine exhibit an anti-inflammatory effect about 20 years ago, and it had already been reported in 'Nature (1953)' about 50 years ago that glucosamine exhibits a cancer cell proliferation-inhibitory effect. However, glucosamine acted on not only cancer cells but also normal cells in in vitro and in vivo experiments. In 2001, when I was jogging, I came up with the idea of adding a function to glucosamine utilizing lectin (galectin, etc.) present in the body, but it was difficult to realize. At that time, analogous disaccharides were expensive (about 40,000 yen/
$25 \mathrm{mg}$ ). Since I could not afford this price to perform cell culture experiments, I had to modify the reagent (disaccharides) to perform this study. I directly made a phone call to the responsible technical manager of the manufacturer, and proposed the possibility of the idea of new sugar chain function. Professional engineers had abundant accumulated knowledge and techniques concerning sugar chain synthesis. Five types of new $\mathrm{di}^{-}$and trisaccharides were sent to me about 2 months later. The names and structures of these were lactosamine $\left(\mathrm{LacNH}_{2}, \mathrm{Gal} 31-4 \mathrm{GlcNH}_{2}\right)$, allolactosamine (AlloLacNH $\mathrm{N}_{2}$, GalB1-6GlcNH${ }_{2}$ ), galactosyllactosamine (GalLacNH ${ }_{2}$, Galß1-4Galß1-4GlcNH${ }_{2}$ ), and UK-1 (unknown structure). I investigated the anti-cancer activity of these saccharides (excluding UK-1 disaccharide) in vitro, but these did not have sufficient activity. The results were far from expected and I was disappointed. The chemical structure of the remaining disaccharide (UK-1) was unclear, but I immediately performed a cancer cell proliferation inhibition experiment with it, while not expecting a good result. After cancer cells were

\footnotetext{
Osamu Hosomi

Graduate School of Health and Sports Science, Juntendo University

1-1 Hirakagakuendai, Inzai-shi, Chiba 270-1695, Japan

TEL: +81-476-98-1001 E-mail: ohosomi@juntendo.ac.jp

[Received Oct. 27, 2015]
} 


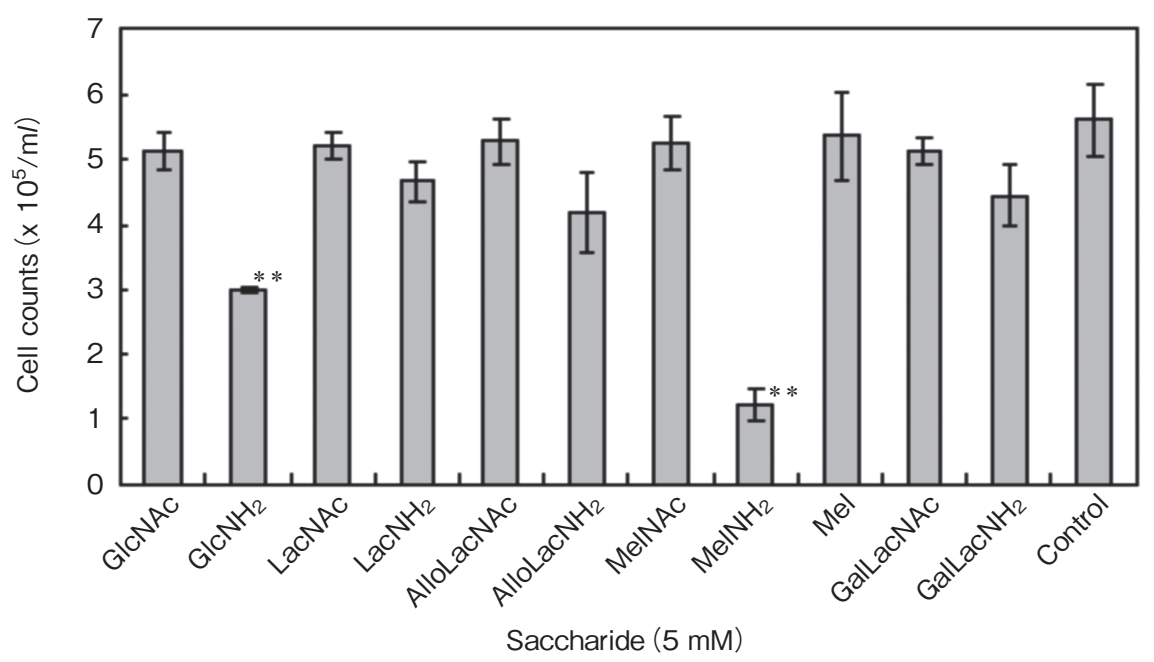

Figure-1 Suppressive effects of saccharides on human leukemia cell (K562) proliferation

GlcNAc: N-acetylglucosamine, GlcNH : glucosamine, LacNAc: N-acetyllactosamine, LacNH${ }_{2}$ : lactosamine, AlloLacNAc: N-acetyl-allolactosamine, AlloLacNH $\mathrm{H}_{2}$ : allolactosamine, MelNAc: $\mathrm{N}$-acetylmelibiosamine (unregistrered), $\mathrm{MelNH}_{2}$ : melibiosamine (unregistered), Mel: melibiose, GalLacNAc: galactosyl-N-acetyllactosamine, GalLacNH $\mathrm{N}_{2}$ : galactosyllactosamine

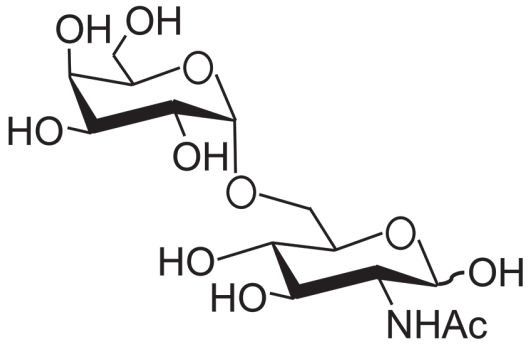

$\mathrm{N}$-acetylmelibiosamine

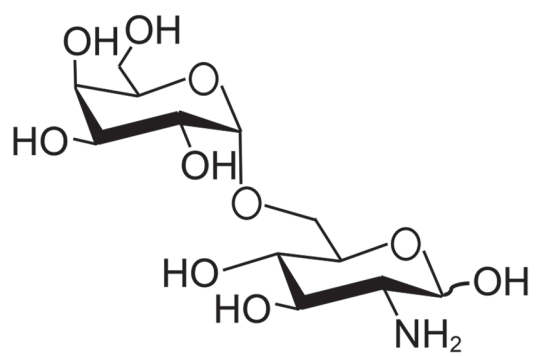

melibiosamine

Figure-2 Structures of novel oligosaccharides

cultured with UK-1 (5 and $10 \mathrm{mM}$ ) for 72 hours, I planned to observe and count viable cancer cells under a microscope. However, not viable cells but only some cell fragments could be observed. I doubted the result and considered that the experiment had failed or that I had forgotten to add cancer cells to culture plate wells. I repeated the experiment in which I carefully and reliably prepared cancer cells and saccharides, and carefully cultured the cells. I repeated the experiment several times, but the results were the same (Figure-1). The structure of this saccharide was identified as $\mathrm{Gal} \alpha 1-6 \mathrm{GlcNH}_{2}$ by NMR analysis (Figure-2), but we had never seen this structure before. I considered various conditions, such as the sample buffer and $\mathrm{pH}$. Considering analogous $\left(\mathrm{Gal}-\mathrm{GlcNH}_{2}\right)$ saccharides, the only difference among these saccharides was glycosidic bonds: $\alpha 1-6, \beta 1-4$, and $\beta 1-6$, and not the constituent sugars. Melibiose (Gal $\alpha 1-6 \mathrm{Glc}$ ) is a natural sugar and similar to the new sugar $\left(\mathrm{Gal} \alpha 1-6 \mathrm{GlcNH}_{2}\right)$. Thus, we arbitrarily named it melibiosamine (Gal $\alpha 1-$ $6 \mathrm{GlcNH}_{2}$ ). We were surprised that this saccharide has not been discovered or synthesized in the world. If we had not used the reverse reaction of glycosidase for sugar synthesis, we would have never come across this new disaccharide, and we were lucky that we did not give up.

From around 2009, my students and I investigated the influence of $\mathrm{MelNH}_{2}$ on several types of cancer cells (colorectal and breast cancers and leukemia), and the effect of $\mathrm{MelNH}_{2}$ was observed in most cells. However, an influence on normal human cells was of concern. We observed it with 
some trepidation, but fortunately, $\mathrm{MelNH}_{2}$ did not affect normal human cells.

While being busy every day, I had to perform most experiments by myself alone, and the experiments did not progress smoothly. We initiated the direct administration of $\mathrm{MelNH}_{2}$ to mice in 2012 . After confirming the size of transplanted cancer tissue, $\mathrm{MelNH}_{2}$ was administered into the cancer tissue only once. About 40 days later, the cancer tissue had disappeared or atrophied. In the second administration experiment, the saccharide was administered to soft tissue around cancer, and the result was the same. Then, we decided to administer $\mathrm{MelNH}_{2}$ by intravenous injection while considering that it may not be effective to inhibit tumor growth because the administration site was too far away to reach cancer tissue. However, the expectation was completely different: cancer tissue disappeared or atrophied in more than $50 \%$ of mice. Furthermore, $\mathrm{MelNH}_{2}$ caused no adverse reaction, and the mice were active. Although $\mathrm{MelNH}_{2}$ was administered to only a small number of mice $(\mathrm{n}=$ 15-20), we expect $\mathrm{MelNH}_{2}$ to be sufficiently effective.

We have to confirm the cancer-inhibitory effect of orally administered $\mathrm{MelNH}_{2}$, for which the mass synthesis of $\mathrm{MelNH}_{2}$ is very important. Fortunately, humans do not have a Gal $\alpha 1-6 \mathrm{GlcNH}_{2}$-digesting enzyme. Therefore, we expect orally ingested $\mathrm{MelNH}_{2}$ to be effective against gastrointestinal cancers. However, it is possible that some human intestinal bacteria have $\alpha$-galactosidase and degrade $\mathrm{MelNH}_{2}$. If this is overcome, absorption of the saccharide from the intestine will become possible. We believe that these expectations for saccharides will be realized in the future.

When I lost confidence and my motivation to continue studying the effect of oligosaccharides on cancer' weakened, I always told myself that it is too early to give it up.

At the end, I am grateful to Juntendo University, friends, students (Figure-3), and my family.

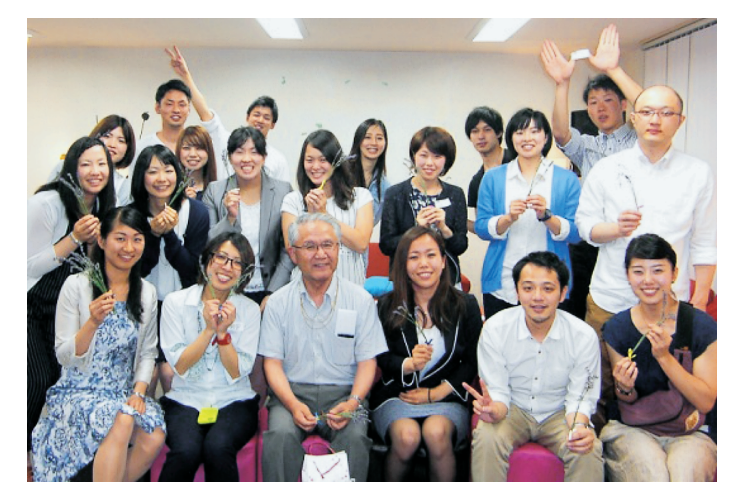

Figure-3 Graduates, graduate students and author (center of front row) 